\title{
PROPERTIES OF DUST IN EARLY-TYPE GALAXIES BASED ON THE ALL-SKY-SURVEY DATA AND NEAR-INFRARED SPECTRA
}

\author{
T. Mori, S. Oyabu, H. Kaneda, D. Ishihara, and M. Yamagishi \\ Graduate School of Science, Nagoya University, Furo-cho, Chikusa-ku, Nagoya, Aichi, 464-8602, Japan \\ E-mail: oyabu@u.phys.nagoya-u.ac.jp \\ (Received July 01, 2012; Accepted August 16, 2012)
}

\begin{abstract}
We present the properties of dust and the near-infrared spectral features in nearby early-type galaxies. The properties of dust are obtained from the AKARI far-infrared all-sky survey diffuse map. The AKARI/IRC is used for the near-infrared spectra. We improve spectral data with the new dark subtraction method on the basis of the knowledge acquired in our laboratory experiments of the engineering-model detector for the IRC. We have succeeded in fitting the continuum by a power-law function and detecting $\mathrm{CO}$ and $\mathrm{SiO}$ absorption features in early-type galaxy spectra. Comparing the properties of dust and near-infrared spectral features, we find that the power-law slope depends on dust temperature, but not on the dust mass, which suggests that low-luminosity AGNs may contribute to the changes in the power-law slope and dust temperature.
\end{abstract}

Key words: galaxies: elliptical and lenticular; ISM: dust; methods: data analysis

\section{INTRODUCTION}

Generally, early-type galaxies are believed to be poor in dust, dominated by old stars and inactive with star formation. However, recent studies suggested that many early-type galaxies have more dust than the expectation, which is determined by the balance between mass loss from evolved stars and sputtering destruction in interstellar hot plasma. The origin of the abundant dust in early-type galaxies is still under discussion. We investigate the relation between dust properties using the far-infrared photometry and spectral features in the near-infrared from the AKARI.

\section{AKARI FAR-INFRARED MAP}

In order to estimate the dust mass and temperature in nearby early-type galaxies, we use the AKARI farinfrared all-sky survey map (Doi et al., in this volume). As a result, many early-type galaxies are found to have a larger amount of dust than the expectation from their optical luminosities (i.e. mass loss rates). We find that their spectral energy distribution is expressed as mul- tiple temperature components and that the dust mass is well correlated with the stellar luminosity which is estimated by the $K_{s}$-band luminosity from the 2MASS.

\section{AKARI NEAR-INFRARED SPECTRA}

To further reveal the nature of the dust, we performed IRC near-infrared spectroscopy with AKARI in the warm phase. For the warm phase data, especially data at detector temperatures $T$ higher than $45 \mathrm{~K}$, we had difficulty in subtracting dark-frame data properly with the official pipeline. We have therefore developed a new method for the dark subtraction procedure.

Our new method is based on our laboratory experiments of the engineering-model detector for the IRC which reveal that the dark current $I_{\text {dark }}$ on each pixel of the detector follows the equation

$$
I_{\text {dark }}=I_{0} \exp \left(-E_{g} / 2 k T\right)
$$

where $k$ is the Boltzmann constant and $I_{0}$ and $E_{g}$ are fitting parameters (Mori et al., 2011). Combining the equation and IRC data, we determined the properties 


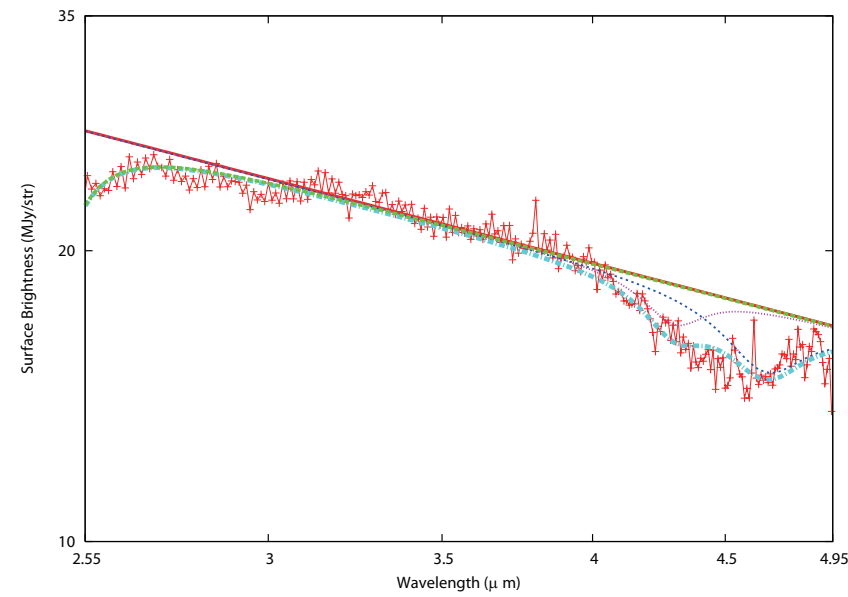

Fig. 1. Example of a spectrum (red) of the early-type galaxy, NGC 1052. The dot-dashed line (cyan) represents a fitted model spectrum which consists of a power-law continuum (thick solid line; red), and $\mathrm{SiO}(\Delta \nu=2)$ and $\mathrm{CO}(\Delta \nu=1)$ absorption features at $4.3 \mu \mathrm{m}$ (dotted line; magenta) and $4.66 \mu \mathrm{m}$ (short-dashed line; blue), respectively. The model also includes the $\mathrm{CO}(\Delta \nu=2)$ absorption at $2.3 \mu \mathrm{m}$ (long-dashed line; green).

for each pixel of the IRC near-infrared detector. The result indicates that the dark current on each pixel is very sensitive to the detector temperature. However, the temperature varies during an observation and the temperature data which is provided by the satellite do not have enough resolution. Thus improper dark subtraction leaves residuals of dark current on the data. Therefore, we searched for accurate temperature information by minimizing the standard deviation of the dark-subtracted data (Ishihara et al., in prep.) and created an accurate dark frame using the temperature and Equation (1). As a result, we have succeeded in improving the spectra. Fig. 1 shows an example of the extracted spectrum.

We detected several absorption features in the nearinfrared spectra. By combining spectra of early-type galaxies at other wavelengths (Vanderbeke et al., 2011; Kaneda et al., 2008), the absorption features are identified as $\mathrm{CO}$ and $\mathrm{SiO}$ bands of late-type stellar photosphere. To parametrize these features, we fit the model which consists of a power-law continuum and absorption features (Fig. 1).

\section{DISCUSSION}

Comparing far-infrared dust properties and nearinfrared spectral features, we find no correlations be-

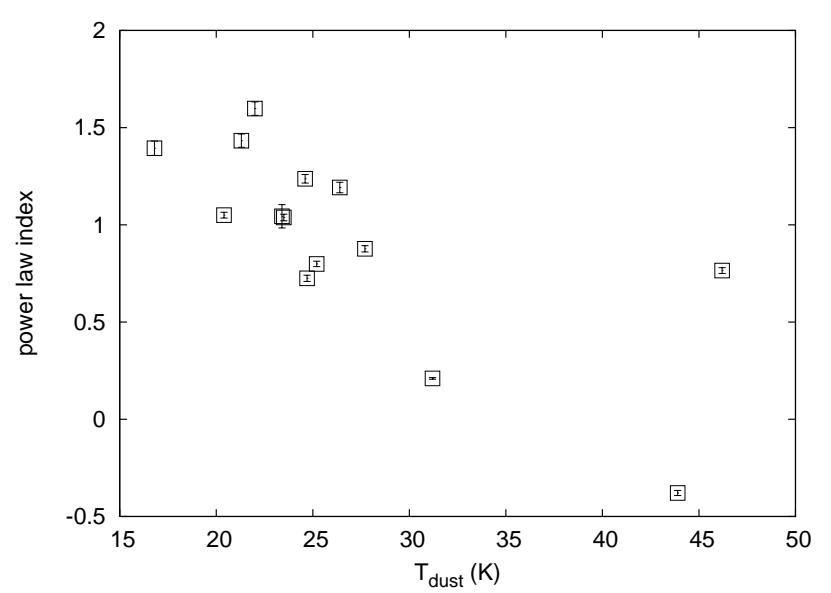

Fig. 2. Power-law index of the continuum emission as a function of the dust temperature.

tween the equivalent width of $\mathrm{CO} / \mathrm{SiO}$ absorption features and dust mass normalized by $K_{\mathrm{S}}$-band luminosity. This indicates that the total dust mass is not related to the current mass loss rate from late-type stars. On the other hand, near-infrared continuum slopes depend on the dust temperatures, as shown in Fig. 2, but not on the dust mass. These results indicate that spectral reddening by dust does not change the continuum slope. Instead, the presence of hot dust changes the slope. Our results suggest that low-luminosity AGNs may contribute to the changes in the continuum slope and dust temperature.

\section{REFERENCES}

Kaneda, H., et al., 2008, Properties of Polycyclic Aromatic Hydrocarbons in Local Elliptical Galaxies Revealed by The Infrared Spectragraph on Spitzer, ApJ, 684, 270

Mori, T., Kaneda, H., Ishihara, D., Oyabu, S., \& Wada, T., 2011, Laboratory and In-Flight Measurements at 10-55 K of InSb Near-Infrared Detector Performance for the Warm Mission of the AKARI Satellite, PASP, 123, 942

Vanderbeke, J., Baes, M., Romanowsky, A., \& Schmidtobreick, L., 2011, Optical and Nearinfrared Velocity Dispersions of Early-type Galaxies, MNRAS, 412, 2017 